\title{
A phylogenetic comparison of gene trees constructed from plastid, mitochondrial and genomic DNA of Plasmodium species
}

\author{
Dharmendar Rathore, Allison M. Wahl, Margery Sullivan, Thomas F. McCutchan* \\ Laboratory of Parasitic Diseases, National Institute of Allergy and Infectious Diseases, National Institutes of Health, \\ 4 Center Drive MSC 0425, Bethesda, MD 20892-0425, USA
}

Received 8 August 2000; accepted in revised form 14 February 2001

\begin{abstract}
Gene trees of Plasmodium species have been reported for the nuclear encoded genes (e.g. the Small Subunit rRNA) and a mitochondrial encoded gene, cytochrome b. Here, we have analyzed a plastid gene coding for caseinolytic protease ClpC, whose structure, function and evolutionary history have been studied in various organisms. This protein possesses a 220-250 amino acid long AAA domain (ATPases associated with a variety of cellular activities) that belongs to the Walker super family of ATPases and GTPases. We have sequenced the AAA motif of this gene, encoding the protein from nine different species of Plasmodium infecting rodents, birds, monkeys, and humans. The codon usage and GC content of each gene were nearly identical in contrast to the widely varying nucleotide composition of genomic DNAs. Phylogenetic trees derived from both DNA and inferred protein sequences have consistent topologies. We have used the $\mathrm{ClpC}$ sequence to analyze the phylogenetic relationship among Plasmodium species and compared it with those derived from mitochondrial and genomic sequences. The results corroborate well with the trees constructed using the mitochondrially encoded cytochrome b. However, an important element distinguishes the trees: the placement of Plasmodium elongatum near the base of the plastid tree, indicating an ancient lineage of parasites in birds that branches from the tree prior to other lineages of avian malaria and the human parasite, P. falciparum. (C) 2001 Elsevier Science B.V. All rights reserved.
\end{abstract}

Keywords: Gene trees; Genomic DNA; Mitochondrial DNA; Phylogenetic comparison; Plasmodium species; Plastid DNA

\section{Introduction}

Plasmodium species possess a plastid-like organelle (the apicoplast) that contains a circular $35 \mathrm{~kb}$ DNA genome resembling the plastid DNA of non-photosynthetic plants [1]. The plastid DNA in Plasmodium is the remnant of an algal plastid genome, probably acquired through secondary endosymbiosis by an ancient progenitor of the phylum [1-3]. The genome is organized in a similar gene order as in the plastids of photosynthetic organisms, except it lacks genes involved in photosynthesis [4]. One central function of the plastid in these obligate intracellular parasites is thought to be its

Abbreviations: AAA, ATPases associated with a variety of cellular activities; Clp, caseinolytic protease; CS, circumsporozoite protein; SSU, small subunit.

* Corresponding author. Tel.: +1-301-4966149; fax: + 1-3014020079.

E-mail address: tmccutchan@niaid.nih.gov (T.F. McCutchan). involvement in the establishment of the parasitophorous vacuole during red cell invasion [5].

The plastid genome encodes several very conserved genes including $\mathrm{ClpC}$, a member belonging to caseinolytic protease family of proteins along with rRNA, tRNA, RNA polymerase subunits and ribosomal proteins [6,7]. The Clp system was first identified as a heat-shock-inducible, multicomponent, ATP-dependent protease complex capable of hydrolyzing casein [8]. ClpC possess a conserved AAA domain (ATPases associated with a variety of cellular activities) that belongs to the Walker super family of ATPases and GTPases (reviewed in Ref. [9]). It has been predicted on the basis of sequence similarity that $\mathrm{ClpC}$ belongs to the double nucleotide binding form of $\mathrm{Clp}$, although only the second of the two ATP-binding domains is conserved in the malarial protein [7].

The ClpC sequence has not previously been used for phylogenetic analysis of the apicomplexa, nor has there 
been a comparison of any plastid-encoded gene for the Plasmodium species. However, the uniparental inheritance of the plastid [10,11], like that of mitochondria, indicates a common and simple history with little or no recombination. Therefore, it represents a more contiguous platform for phylogenetic analysis. Our study has included nine different species of Plasmodium infecting rodents, birds, monkeys and humans. This is the first report in which a sequence from the plastid has been utilized for exploring the phylogenetic relationship that exists between various Plasmodium species.

\section{Materials and methods}

\subsection{Reagents}

Reagents for isolation of DNA were obtained from Life-Technologies Inc. (Gaithersburg, MD). Reagents for PCR were obtained from Perkin Elmer Cetus Corporation (Emeryville, CA). The TA cloning kit was purchased from Invitrogen (San Diego, CA). The Geneclean spin kit was obtained from BIO101 (Carlsbad, CA).

\subsection{Preparation of total parasite $D N A$}

Infected blood samples were used as source to isolate parasite DNA from nine species of Plasmodium. Complete DNA preparations of different species of the Plasmodium parasite was prepared according to the protocol of Trager [12]. Briefly, red cells were lysed with $0.15 \%$ saponin, and intact parasite cells were isolated. These parasites were then used to isolate total DNA by proteinase $\mathrm{K}$ digestion and RNase treatment.

\subsection{PCR amplification of ClpC fragment}

The AAA motif was amplified by PCR from total genomic DNA using two primers (1421 and 1423) designed from the published $P$. falciparum $\mathrm{ClpC}$ sequence available at the NCBI database (Accession number X95276). Primers 1421 5'AAAACTGAATTAGCAAAAATATTA $3^{\prime}$ and 1423 5'ATCCTTTATATGGAGCTCG 3' yielded a product of approximately 640 bp. A 30-cycle PCR reaction was performed with denaturation, annealing and amplification for $1 \mathrm{~min}$ each at 94,45 and $62^{\circ} \mathrm{C}$, respectively. PCR products were separated by electrophoresis in a $1 \%$ agarose gel, excised from the gel and purified using the Gene Clean Kit.

\subsection{Cloning and sequencing of ClpC fragment}

Purified PCR products were cloned using a TOPO TA vector cloning kit as suggested by the manufac- turer, Invitrogen (San Diego, CA). Clones were sequenced in complementary directions using $\mathrm{T} 7$ and Universal reverse oligos binding in the vector. The sequences have been submitted to GenBank with accession numbers AF348337 ( $P$. berghei), AF348338 ( $P$. cynomolgi), AF348339 (P. elongatum), AF348340 ( $P$. gallinaceum), AF348341 ( $P$. knowlesi), AF348342 ( $P$. malariae), AF348343 (P. relictum) and AF348344 ( $P$. vivax).

\subsection{Phylogenetic analysis of $\mathrm{ClpC}$ sequences}

The DNA sequences obtained from the eight species of Plasmodium were translated into amino acid sequences and analyzed for AT content and codon usage with the Edit Seq program of the DNASTAR package. The ClpC DNA sequence of Plasmodium falciparum (Accession number X95276) and Toxoplasma gondii (accession number U87145) used as an outgroup, were obtained from GenBank. The DNA and amino acid sequences were aligned using Clustal $\mathrm{W}$ in the Megalign program of the DNASTAR package. The tree was constructed using the neighbor-joining method [13] in PAUP version 4.0b3a [14].

\section{Results and discussion}

We have utilized the AAA motif of $\mathrm{ClpC}$ protein to analyze the phylogenetic relationship of nine different species of malaria parasites and to compare this tree with trees derived from genes originating in the mitochondria and nucleus. DNA encoding AAA motif was amplified by PCR utilizing primers designed on the basis of previously published $P$. falciparum $\mathrm{ClpC}$ sequence [7]. Multiple clones were sequenced for each species on an automated sequencer to minimize errors in subsequent phylogenetic analysis. Fig. 1 depicts the alignment, using Clustal W, of the AAA domains of the $\mathrm{ClpC}$ protein. The high degree of gene conservation and the lack of significant differences in the GC content or pattern of codon usage led to a solid alignment that did not appear to have ambiguities. Phylogenetic relationships were inferred by maximum parsimony, neighbor-joining and distance (Kimura's two-parameter model) using PAUP version 4.0b3a. All yielded trees with identical topologies. The results are shown as neighbor-joining trees because that allows a direct comparison with the published data of Escalante and Lal, who analyzed genes from many of the same organisms. We have chosen Toxoplasma gondii as an outgroup because studies by Egea and Lang-Unnasch have demonstrated, using sequence data from the small subunit rRNA of other Apicomplexa, that Toxoplasma gondii branches just prior to the stem that gives rise to Plasmodium species [15]. More outgroups are important 
in establishing solid relationships between the organisms [16] and were used in constructing the protein tree (see below).

To compare our plastid-based tree with Plasmodium trees from other sources, we constructed two parallel trees using sequences that were of mitochondrial (cytochrome b, Fig. 2b) [17] and genomic (SSU rRNA, Fig. 2c) $[18,19]$ origin. The same species of Plasmodium $[18,20,21]$ were used in both trees with the following exceptions. $P$. relictum was not available for the cytochrome $\mathrm{b}$ tree, and $P$. elongatum is not available for the ribosomal RNA tree. P. lophurae was included in the SSU rRNA-based tree to maintain the same relative ratios of $\mathrm{A}-\mathrm{T}$-rich sequences giving a topology that is the same as originally published.

Phylogenetic analysis of the ClpC DNA sequences showed that the avian parasite $P$. elongatum was first to diverge from the Plasmodium stalk, suggesting that its progenitor was among the most ancient forms of malaria (Fig. 2a). The origin of P. elongatum is wellsupported with a $100 \%$ bootstrap score suggesting that a progenitor of both rodent and P. falciparum was an avian parasite. Although the stem later bifurcates giving rise to separate rodent and primate malaria lineages, the exact placement of the node is in question because of a low bootstrap support (31\%). (The corresponding node in the cytochrome $\mathrm{b}$ derived tree is positioned differently and has a more significant bootstrap value.) The branch that gives rise to $P$. falciparum is the first to diverge from the primate stalk as in the case with the cytochrome $b$ tree. This does not necessarily indicate that the progenitor itself was the human parasite, $P$. falciparum, when it diverged from the main stock of the tree. It does indicate that sometime between the divergence and the establishment of presentday infectious agent of humans, a switch in hosts occurred. The branching order of simian and human parasites is consistent with the previously published reports utilizing small subunit rRNA [18,20,21], circumsporozoite protein $[22,23]$ and cytochrome $\mathrm{b}$ sequences [17]. The relationship of $P$. cynomolgi with $P$. vivax, however, is consistent with the trees derived from genomic sources but somewhat different from that found in the cytochrome $b$ tree.
1 KTELAKILSKQLFGSEKELIRFDMSEYMEKHSISRLIGSPPGYVGYSEGGQLTE 54

$\ldots \ldots \ldots . \ldots \ldots \ldots \ldots \ldots \ldots \ldots \ldots$

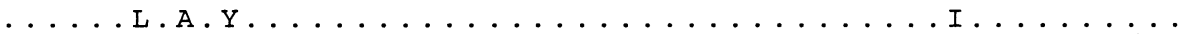

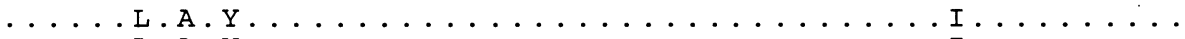

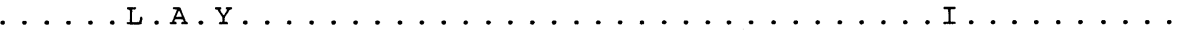

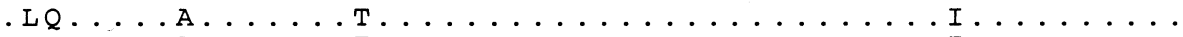

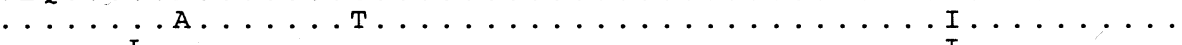

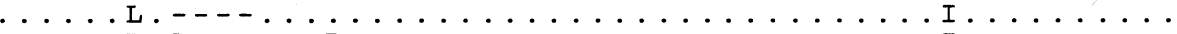

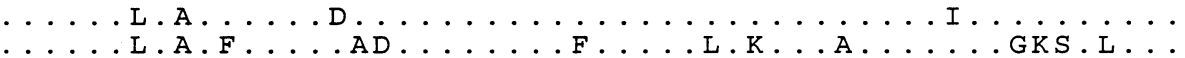

55 QVYKKPNSVILFDEIEKAHPDIYNIMLQILDEGRLTDSTGKLIDFTHTIILLTS 108

$\ldots N \ldots$. . . . . . . . . . . . . . . . . . .

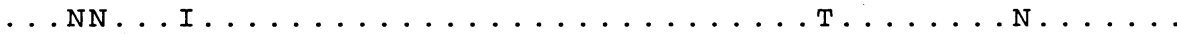

$\ldots$. . . I . . . . . . . . . . . . . . . . . . . . .

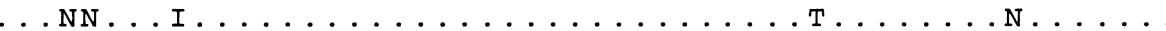

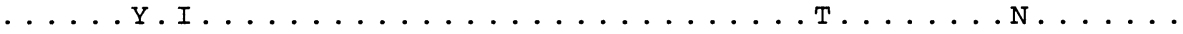

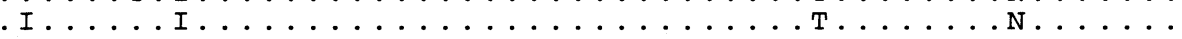

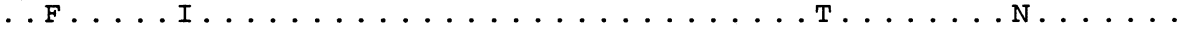

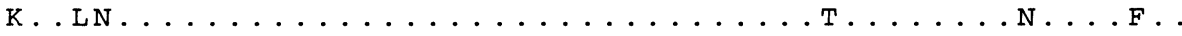

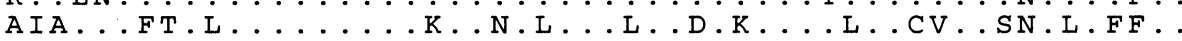

109 NLGCPKN-YDL-YLKNKNFLSKSDLKEIEKNIKININNYFKPELLNRLTNILIF 160

$\ldots \ldots T .-\ldots K-\ldots N \ldots Y$. . . . . D . KN . . SK.T. . . . . . .

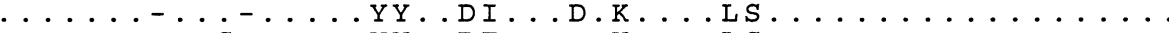

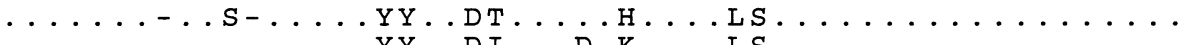

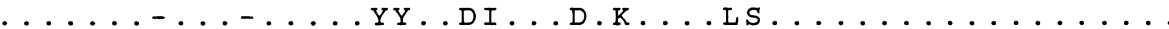

$\ldots \ldots \ldots . \ldots \mathrm{I}-\ldots \ldots \mathrm{DY} \ldots \mathrm{NF} \ldots \mathrm{EQ} . \mathrm{N} \ldots \mathrm{L} \ldots \ldots \ldots$

$\ldots \ldots \mathrm{D}-\mathrm{N}_{\mathrm{I}}-\ldots \mathrm{T} \ldots \mathrm{Y} \ldots \mathrm{NL} \ldots \mathrm{K} \ldots \ldots \mathrm{LY} \ldots \ldots \ldots$

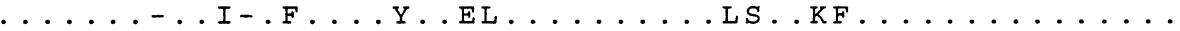

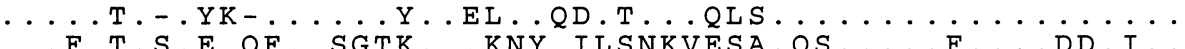

161 NPLNINNLLFIFNKF INELKIKLYLNKLNIIIHINKELKYFLVKLMYNPLYGA 213

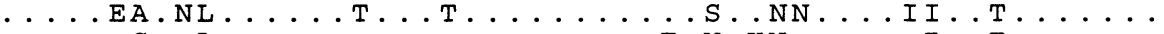

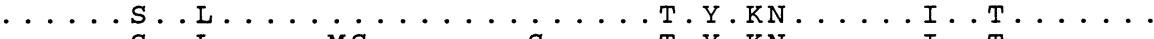

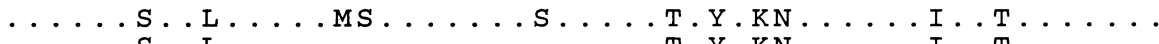

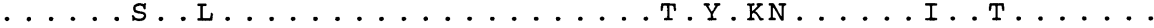

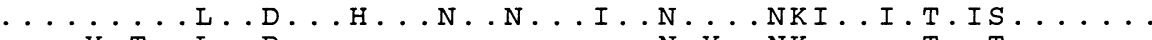

$\ldots \mathrm{Y} . \mathrm{T} \ldots \mathrm{L} \ldots \ldots \ldots \ldots \mathrm{N} . \mathrm{NK} \ldots \mathrm{N} \ldots \mathrm{T} \ldots \ldots$

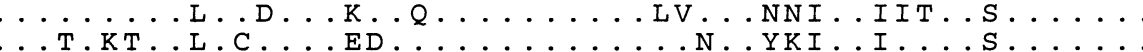

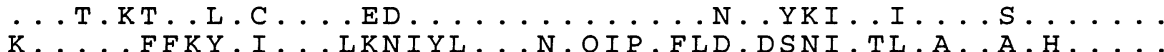

P. falciparum

$P$. berghei

P. cynomolgi

$P$. knowlesi

P. Vivax

$P$. relictum

$P$. malariae

P. gallinaceum

P. elongatum

$T$. gondii

P. falciparum

$P$. berghei

P. cynomolgi

P. knowlesi

P. Vivax

P. relictum

P. malariae

P. gallinaceum

$P$. elongatum

T. gondii

P. falciparum
$P$. berghei
$P$. cynomolgi
$P$. knowlesi
$P$. vivax
P. relictum
P. malariae
$P$. gallinaceum
$P$. elongatum
T. gondii
P. falciparum
$P$. berghei
$P$. cynomolgi
$P$. knowlesi
$P$. vivax
$P$. relictum
$P$. malariae
$P$. gallinaceum
$P$. elongatum
T. gondii

Fig. 1. Alignment of AAA motif of ClpC protein sequences using CLUSTAL W. Only residues that differ from $P$. falciparum ClpC sequence have been shown. Identical residues are marked as a dot (.), while gaps in alignment have been marked with a dash (-). 
ClpC

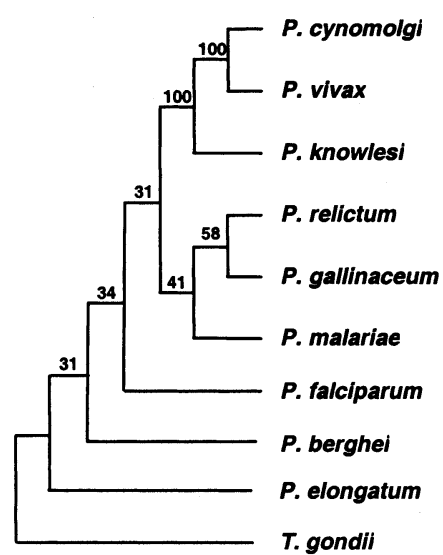

Cytochrome b

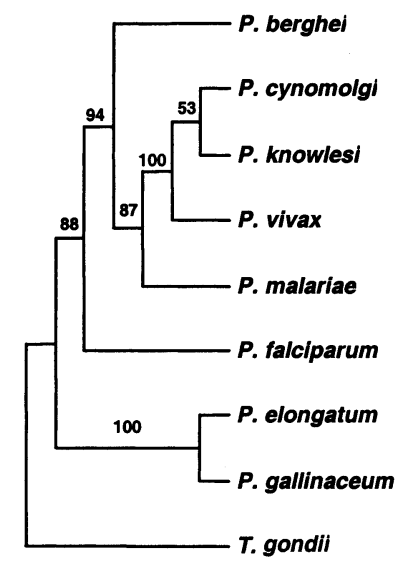

SSU

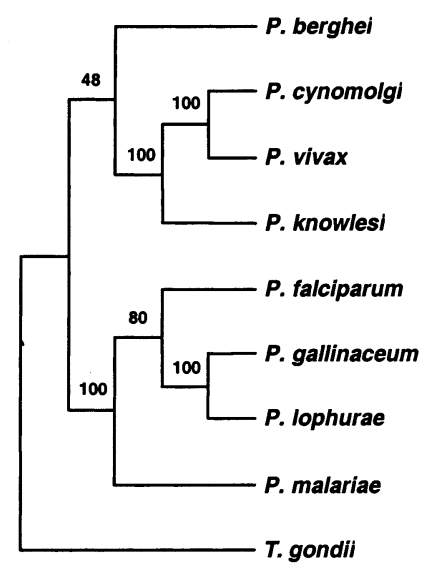

Fig. 2. Phylogenetic analysis of nine different species of Plasmodium. Neighbor-joining trees of Plasmodium species utilizing ClpC, cytochrome $\mathrm{b}$ and the small subunit rRNA sequences. The bootstrap values are shown on the branches and indicate the number of times out of 1000 replications that the species to the right of the branch appear as a clade. Toxoplasma gondii was used as an outgroup to root the tree.

A tree was also derived using the same algorithms for the corresponding protein sequence. The topology of the tree derived from protein sequence of $\mathrm{ClpC}$ was done using both algal and chloroplast outgroups in addition to Toxoplasma gondii, and had an identical topology to that derived from the nucleic acid sequence (data not shown). Previously, Barta et al. have demonstrated that an outgroup composed of ciliates and of dinoflagellates provides the most robust phylogenetic estimate of relationships among apicomplexan taxa [24].

The placement of $P$. elongatum at the base of the ClpC tree supports the idea that the progenitor of $P$. elongatum appeared earlier than the other parasites under examination. The $\mathrm{ClpC}$ tree also differs from the other trees in that $P$. falciparum comes after one avian parasite, $P$. elongatum, but before another, $P$. relictum. The topology of the ClpC tree would appear to support the criteria suggested by Brooks and McLennan [25] needed to show lateral transfer, while the topology of ribosomal RNA and cytochrome $b$ trees does not. Whether more data will support and hence strengthen these relationships is an open question. Parenthetically, some do not favor the idea of lateral transfers on the grounds that it implies both a switch in primary and secondary hosts. This is, however, not a ground to reject the idea in that although mosquito species do have preferred sources of blood meals, they do not restrict their search for blood if that species is not available. For example, Aedes and Culex mosquitoes are the most common host of avian parasites and seem to prefer the blood of birds, but humans are constantly bitten by both. Anopheles mosquitoes, which carry human malaria certainly can serve as a host for avian malaria. In fact it has recently been shown that sporozoites can develop, even in Drosophila [26]. Further, in our opinion, the contact of humans with avian parasites is not so rare. The interaction simply does not manifest itself as a serious medical concern. A parallel may be drawn with schistosomes of birds that occasionally cause "swimmer's itch" in humans.

It might be expected that the topologies of trees created using the same algorithms, DNA sources and outgroups, as described here, would have the same topologies, even if branch lengths differed. They do not in the case described here. The difference in topologies could be due to artifactual errors inherent in the analysis or differences in events occurring in the history of the individual genes. Hence, one is obligated to describe potential sources of error, which could distort relationships when publishing phylogenetic relationships $[27,28]$. Enea and coworkers have pointed out potential problems with the use of rRNA to infer Plasmodium phylogeny $[29,30]$. Selective pressure on the sequence of surface antigens, as indicated, for example, by the strong bias of non-synonymous to synonymous mutations [31], may have an effect on their use for the construction of phylogenetic trees. The distribution of the mutations in both $\mathrm{Clp} \mathrm{C}$ and cytochrome $\mathrm{b}$ shows a more standard type of variation with synonymous mutations being predominant. Hence, it is less likely that selection plays a significant role in its sequence variability.

GC content and codon usage bias can incorrectly sway the topology of a phylogenetic tree. This is especially problematic in analyzing Plasmodium species because the AT content varies widely in the genus (Table 1). P. falciparum has the lowest GC content, $26 \%$ in its 
coding sequences, while $P$. vivax genes show a balanced usage with a GC content of $48 \%$, although both species infect humans (Table 1). Although there are approaches for compensating for this type of variation, it can still lead to serious ambiguity. Unlike plants, where GC content in both genomic and chloroplast coding sequences is comparable (Table 1), a careful look at the $\mathrm{GC}$ content of the $\mathrm{ClpC}$ sequences in Plasmodium revealed that all had GC contents clustering around $19 \%$ (Table 1). In comparing the codon usage within the $\mathrm{ClpC}$ sequence with the codon usage of the entire plastid genome, it seems that the AT content of $\mathrm{ClpC}$ is representative of the entire plastid. Codons not found in the AAA motif of $\mathrm{ClpC}$ are also absent or occurred at extremely low frequencies in the plastid genome (data not shown). The constancy of the GC content and codon usage of $\mathrm{ClpC}$ sequences across the genus is of tremendous advantage in establishing the phylogenetic relationships. Extreme AT richness of the plastid genome sequences across the species suggests that the plastid has maintained its codon usage preferences independent of the usage of the genome. This bias could be related to the minimal content (number) of transfer RNAs encoded within the plastid genome and the manner and extent to which the "wobble" phenomenon affects translation in the plastid. It is clear from this work that the constraints on sequence change are different from those found in the genome. AT richness of the plastid genome could be a characteristic feature of apicomplexa as the GC content in T. gondii genomic and plastid sequences is 53 and $16 \%$, respectively.

An increasing awareness of the transfer of genetic material between organisms suggests that inferences derived from a single gene tree may not accurately reflect the true phylogenetic relationship of organisms. It is important to emphasize that no one tree may best

Table 1

Comparison of the GC content of the genomic coding sequences versus $\mathrm{ClpC}$ sequence in different species of Plasmodium

\begin{tabular}{llll}
\hline Species & \multirow{2}{*}{ Host } & \multicolumn{2}{l}{ GC content $(\%)$} \\
\cline { 3 - 4 } & & Genomic & ClpC sequence \\
\hline P. falciparum & Human & 25.87 & 17.45 \\
$P$. vivax & Human & 47.97 & 18.80 \\
$P$. malariae & Human & 39.90 & 16.82 \\
$P$. knowlesi & Monkey & 39.93 & 20.15 \\
$P$. cynomolgi & Monkey & 43.74 & 18.80 \\
$P$. elongatum & Avian & & 18.95 \\
$P$. relictum & Avian & & 19.95 \\
$P$. gallinaceum & Avian & 33.17 & 18.38 \\
$P$. berghei & Rodent & 31.06 & 19.70 \\
T. gondii & Human & 53.02 & $16.37^{\mathrm{a}}$ \\
$P$. sativum & & 43.57 & $39.65^{\mathrm{a}}$ \\
C. caldarium & & 43.42 & $33.10^{\mathrm{a}}$ \\
& & & \\
\end{tabular}

${ }^{\text {a }} \mathrm{GC}$ content in whole plastid coding sequences. reflect the history of the organism and that the differences may be indicative of a history that we do not yet fully understand. In other words, the gene tree is representative of the history of the gene, not necessarily the organism. A survey of multiple genes of both genomic and organellar origin should provide an increasingly more defined view of the relationship among organisms, even if all relationships do not yield the same tree-like topology. The data from three interrelated but independent sources of malaria genes yield trees that are largely consistent in topology but with a few interesting exceptions.

\section{References}

[1] Williamson DH, Gardner MJ, Preiser P, Moore DJ, Rangachari $\mathrm{K}$, Wilson RJ. The evolutionary origin of the $35 \mathrm{~kb}$ circular DNA of Plasmodium falciparum: new evidence supports a possible rhodophyte ancestry. Mol Gen Genet 1994;243:249-52.

[2] Kohler S, Delwiche CF, Denny PW, Tilney LG, Webster P, Wilson RJ, Palmer JD, Roos DS. A plastid of probable green algal origin in Apicomplexan parasites [see comments]. Science 1997;275:1485-9.

[3] Wilson RJ, Williamson DH. Extrachromosomal DNA in the Apicomplexa. Microbiol Mol Biol Rev 1997;61:1-16.

[4] Feagin JE. The extrachromosomal DNAs of apicomplexan parasites. Annu Rev Microbiol 1994;48:81-104.

[5] Fichera ME, Roos DS. A plastid organelle as a drug target in apicomplexan parasites. Nature 1997;390:407-9.

[6] Gardner MJ, Feagin JE, Moore DJ, Rangachari K, Williamson $\mathrm{DH}$, Wilson RJ. Sequence and organization of large subunit rRNA genes from the extrachromosomal $35 \mathrm{~kb}$ circular DNA of the malaria parasite Plasmodium falciparum. Nucleic Acids Res 1993;21:1067-71.

[7] Wilson RJ, Denny PW, Preiser PR, Rangachari K, Roberts K, Roy A, Whyte A, Strath M, Moore DJ, Moore PW, Williamson DH. Complete gene map of the plastid-like DNA of the malaria parasite Plasmodium falciparum. J Mol Biol 1996;261:155-72.

[8] Hwang BJ, Park WJ, Chung CH, Goldberg AL. Escherichia coli contains a soluble ATP-dependent protease (Ti) distinct from protease La. Proc Natl Acad Sci USA 1987;84:5550-4.

[9] Beyer A. Sequence analysis of the AAA protein family. Prot Sci 1997;6:2043-58.

[10] Creasey A, Mendis K, Carlton J, Williamson D, Wilson I, Carter R. Maternal inheritance of extrachromosomal DNA in malaria parasites. Mol Biochem Parasitol 1994;65:95-8.

[11] Vaidya AB, Morrisey J, Plowe CV, Kaslow DC, Wellems TE. Unidirectional dominance of cytoplasmic inheritance in two genetic crosses of Plasmodium falciparum. Mol Cell Biol 1993;13:7349-57.

[12] Trager W. Malaria parasites (Plasmodium lophurae) developing extracellularly in vitro: incorporation of labeled precursors. J Protozool 1971;18:392-9.

[13] Saitou N, Nei M. The neighbor-joining method: a new method for reconstructing phylogenetic trees. Mol Biol Evol 1987;4:40625.

[14] Swofford DL. PAUP* Phylogenetic Analysis Using Parsimony and Other Methods. Sunderland, MA: Sinauer Associates, 1998.

[15] Egea N, Lang-Unnasch N. Phylogeny of the large extrachromosomal DNA of organisms in the phylum Apicomplexa. J Eukaryot Microbiol 1995;42:679-84 published erratum appears in J. Eukaryot. Microbiol. 1996 Mar-Apr;43(2):158. 
[16] Siddall ME, Barta JR. Phylogeny of Plasmodium species: estimation and inference. J Parasitol 1992;78:567-8.

[17] Escalante AA, Freeland DE, Collins WE, Lal AA. The evolution of primate malaria parasites based on the gene encoding cytochrome $b$ from the linear mitochondrial genome. Proc Natl Acad Sci USA 1998;95:8124-9.

[18] Waters AP, Higgins DG, McCutchan TF. Plasmodium falciparum appears to have arisen as a result of lateral transfer between avian and human hosts. Proc Natl Acad Sci USA 1991;88:3140-4.

[19] Waters AP, Higgins DG, McCutchan TF. Evolutionary relatedness of some primate models of Plasmodium. Mol Biol Evol 1993;10:914-23.

[20] Escalante AA, Ayala FJ. Phylogeny of the malarial genus Plasmodium, derived from rRNA gene sequences. Proc Natl Acad Sci USA 1994;91:11373-7.

[21] Escalante AA, Goldman IF, De Rijk P, De Wachter R, Collins WE, Qari SH, Lal AA. Phylogenetic study of the genus Plasmodium based on the secondary structure-based alignment of the small subunit ribosomal RNA. Mol Biochem Parasitol 1997;90:317-21.

[22] McCutchan TF, Kissinger JC, Touray MG, Rogers MJ, Li J, Sullivan M, Braga EM, Krettli AU, Miller LH. Comparison of circumsporozoite proteins from avian and mammalian malarias: biological and phylogenetic implications. Proc Natl Acad Sci USA 1996;93:11889-94.
[23] Escalante AA, Barrio E, Ayala FJ. Evolutionary origin of human and primate malarias: evidence from the circumsporozoite protein gene. Mol Biol Evol 1995;12:616-26.

[24] Barta JR, Jenkins MC, Danforth HD. Evolutionary relationships of avian Eimeria species among other Apicomplexan protozoa: monophyly of the apicomplexa is supported. Mol Biol Evol 1991;8:345-55.

[25] Brooks DR, McLennan DA. The evolutionary origin of Plasmodium falciparum. J Parasitol 1992;78:564-6.

[26] Schneider D, Shahabuddin M. Malaria parasite development in a Drosophila model. Science 2000;288:2376-9 in process citation.

[27] Grundy WN, Naylor GJ. Phylogenetic inference from conserved sites alignments. J Exp Zool 1999;285:128-39.

[28] Bickel DR. Implications of fluctuations in substitution rates: impact on the uncertainty of branch lengths and on relative-rate tests. J Mol Evol 2000;50:381-90.

[29] Enea V, Corredor V. The evolution of plasmodial stage-specific rRNA genes is dominated by gene conversion. J Mol Evol 1991;32:183-6.

[30] Li J, Collins WE, Wirtz RA, Rathore D, Lal AA, McCutchan TF. Geographic subdivisions of the range of the malaria parasite Plasmodium vivax. Emerg Infect Dis 2001;7:35-42.

[31] de la Cruz VF, Lal AA, McCutchan TF. Sequence variation in putative functional domains of the circumsporozoite protein of Plasmodium falciparum. Implications for vaccine development. J Biol Chem 1987;262:11935-9. 